\title{
Article \\ Photodetector Fast Spectral Response Estimation Using Black Body Radiation
}

\author{
Riccardo Maria Liberati ${ }^{1, t, \ddagger},{ }^{*}$,Alessio Del Paggio ${ }^{2, \ddagger}$, Massimiliano Rossi ${ }^{3, t, \ddagger(D)}$ \\ 1 MBDA Italia S.p.A. Via Monte Flavio, 45 - 00131- Roma - Italy; riccardo.liberati@mbda.it \\ 2 University of Cassino and Southerm Lazio, Cassino(FR), Via G. Di Biasio, 43; \\ alessio.del-paggio@external.mbda.it \\ 3 MBDA Italia S.p.A. Via Monte Flavio, 45 - 00131- Roma - Italy; massimiliano.rossi@mbda.it \\ * Correspondence: riccardo.liberati@mbda.it; Tel.: +39-06-87711 \\ $\ddagger$ These authors contributed equally to this work.
}

\begin{abstract}
We propose a method for the estimation of the spectral response of a photodetector, using only the variation of the temperature of a black body source without the need of an expensive monochromator or a circular filter. The proposed method is suitable especially for infrared detectors in which the cut - off wavelength and the responsivity vs. wavelength is not exactly known. The method provides a rough estimation of the curve $S(\lambda)$ solving a Fredholm integral equation of the first kind. The precision of this technique depends on the number of temperatures at which the detector output is measured. Some example is given in order to better explain the proposed technique.
\end{abstract}

Keywords: Infrared; Photodetector; Black Body

\section{Introduction}

Infrared photodetectors are currently used in many applications. One of the most promising field of application is the passive detection of infrared radiation coming from objects in a given field of view. In order to design an infrared passive system is mandatory to know the parameters characterizing the detector. For the staring detectors the NETD (Noise-equivalent temperature) is very important because it gives information about the sensitivity of the 2D (two-dimensional) array. Another important parameter is the Responsivity. Responsivity can be defined as the ratio between the electrical signal of the detector and the incident optical power. The black body responsivity is defined as the ratio of the signal (voltage or current) and the total power emitted by a black body:

$$
R_{b b}=\frac{V}{\sigma T^{4}}
$$

Unfortunately, in the case of real detectors, only a little amount of this power is 'seen' by the detector and in particular in the case of photonic detectors, only the power between zero wavelength and cut off wavelength creates electron - hole pairs. So the real responsivity is the ratio between the electrical signal and the power given by:

$$
J=\int_{0}^{\infty} W_{\lambda, T} S(\lambda) d \lambda
$$

Where

- $W_{\lambda, T}$ :Planck radiation law

- $S(\lambda)$ : spectral response of the detector

$$
W_{\lambda, T}=\frac{C_{1}}{\lambda^{5}} \frac{1}{\exp \left(\frac{C_{2}}{\lambda T}\right)-1}
$$


where $C_{1}$ and $C_{2}$ are given coefficient. When the detector is made using a single element semiconductor like Silicon or Germanium, the cut - off wavelength and the spectral are known with a little error. Also in the case of two elements alloys like InSb the cut - off wavelength can be considered known and so the $S(\lambda)$. The case of HCT, for example, is quite different because the cut - off wavelength and the spectral response function $S(\lambda)$ could be unknown if we don't know the molar ratio of $\mathrm{HgTe}$ and $C d T e$. Taking into account this problem, it is very important to measure or to estimate the function $S(\lambda)$. The best method is to use a monochromator in order to measure it exactly. For a rough estimate of this quantity we propose a method based only on the variation of the temperature of a calibrated black body.

\section{Results}

The study have been conducted considering the black-body as a radiating source. The black-body radiation power is given by:

$$
W_{\lambda, T}=\frac{C_{1}}{\lambda^{5}} \frac{1}{\exp \left(\frac{C_{2}}{\lambda T}\right)-1}
$$

where:

- $\lambda$ as the wavelength in $\mu \mathrm{m}$

- $\mathrm{T}$ the absolute temperature in Kelvin

- $\mathrm{C} 1$ and $\mathrm{C} 2$ as Kirchoff constants (C1=37418, C2=14388).

The radial spectral density curve, calculated, for example, at $500 \mathrm{~K}$ is reported in Figure 1 . Integrating the Plank function between $\lambda=0$ and $\lambda=\infty$, it's possible to calculate the incident power on a photodetector as:

$$
P=\frac{\sigma T^{4}}{\pi} \frac{\pi r^{2}}{L^{2}} \pi r_{0}^{2}
$$

where:

- $T$ is the absolute temperature in $\mathrm{K}$

- $r$ is the radius of the black-body aperture in $\mu \mathrm{m}$

- $r_{0}$ is the radius of the receiver's optical in $\mu \mathrm{m}$

- $L$ is the distance between the black-body and the photodetector in $\mathrm{cm}$

- $\sigma T^{4}$ is computed from Stefan Boltzman formula:

$$
\sigma T^{4}=\int_{0}^{\infty} W_{\lambda, T} d \lambda
$$

Given the incident optical power on the photodetector it's possible to compute the black-body

responsivity as the ratio between the photodetector output voltage (or current) and the optical incident power:

$$
R_{b b}=\frac{V}{P}
$$

The previous formula doesn't take in to account the spectral response of the photodetector that increases until the cut-off frequency, where it falls to zero. In order to know the exact amount of optical power seen by the detector it's very important to know the spectral response of the detector. In general, this function can be obtained using optical devices like filters or monochromators. The peak responsivity $R_{\lambda}$, which is a function of the spectral response of the photodetector is:

$$
R_{\lambda}=\frac{J(T)}{\pi} \frac{\pi r^{2}}{L^{2}} \pi r_{0}^{2}
$$

Where $J(T)$ is:

$$
J(T)=\int_{0}^{\infty} W_{\lambda, T} S(\lambda) d \lambda
$$




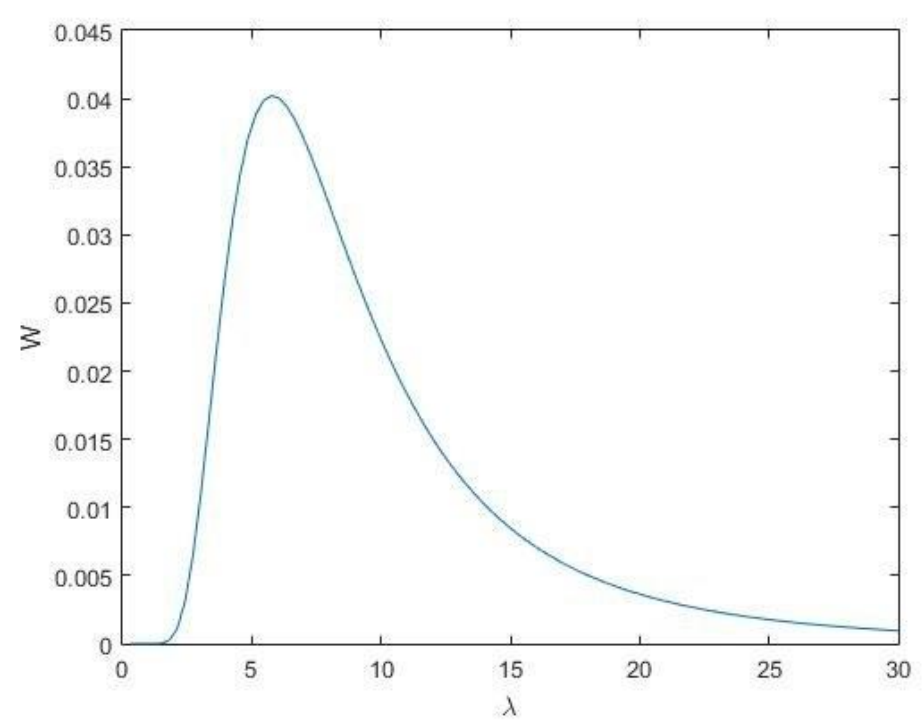

Figure 1. Energy density curve for $T=500 \mathrm{~K}$

From 9 it's possible to compute the photodetector peak responsivity taking into account the function $J(T)$. For this purpose, considering the expression:

$$
V(T)=\frac{\pi r_{0}^{2} r^{2} R_{\lambda}}{L^{2}} J(T)
$$

and defining $\beta$ as:

$$
\beta=\frac{\pi r_{0}^{2} r^{2} R_{\lambda}}{L^{2}}
$$

Then the photodetector output voltage is proportional to the function $J(T)$ by the constant $\beta$. Observing the expression of $J(T)$, it's possible to note that the upper integration limit coincides with the sensor's cut-off wavelength because there is no response above the cut-off frequency. So, the expression for $J(T)$ can be put as:

$$
J(T)=\int_{0}^{\lambda_{c o}} W_{\lambda, T} S(\lambda) d \lambda
$$

The equation is tied to the temperature so that, in general, there will be an integral function for each temperature $T_{j}$ :

$$
V\left(T_{j}\right)=\beta \int_{0}^{\lambda_{c o}} W_{\lambda, T_{j}} S(\lambda) d \lambda
$$

This is the known as the Fredholm integral of the first kind where the unknown function is the spectral response $S(\lambda)$. There are a lot of methods to solve a integral equations in literature and every method has advantages and disadvantages.

\section{Generality of the problem}

Starting from the Fredholm integral of the first kind, it's necessary to find out a method for estimation of the unknown function $f(t)$ :

$$
g(x)=\int_{a}^{b} h(x, t) f(t) d t
$$

The generic function $f(x)$ can be expressed as a series of functions where $\phi(x, k)$ are the basis functions: 


$$
f(x)=\sum_{k=0}^{N} a_{k} \phi(x, k)
$$

The integral will be:

$$
g\left(x_{k}\right)=\sum_{k=0}^{N} a_{k} \int a^{b} h\left(x_{k}, t\right) \phi(x, k) d t
$$

Now it's possible to use the matrix formalism in order to obtain:

$$
\left(\begin{array}{c}
g\left(x_{0}\right) \\
\vdots \\
g\left(x_{N}\right)
\end{array}\right)=\left(\begin{array}{ccc}
J_{0}\left(x_{0}\right) & \ldots & J_{N}\left(x_{0}\right) \\
\vdots & \ddots & \vdots \\
J_{0}\left(x_{0}\right) & \ldots & J_{N}\left(x_{N}\right)
\end{array}\right)\left(\begin{array}{c}
a_{0} \\
\vdots \\
a_{N}
\end{array}\right)
$$

where:

$$
J_{j}\left(x_{k}\right)=\int_{a}^{b} h\left(x_{k}, t\right) \phi\left(x_{j}, t\right) d t
$$

So, in a compact representation, the vector $g(x)$ is the product of the matrix $J(T)$ and the coefficients vector a:

$$
g=J a
$$

From (19) it's possible to calculate the coefficients $a$ :

$$
a=J^{-1} g
$$

Once computed all the coefficients, it's possible to reconstruct the unknown function $f(t)$.

\section{Estimation with basis function}

In the proposed work some basis functions have been identified for the approximation of the spectral response of photodetectors:

- Power series: where the spectral response is estimated as:

$$
S(\lambda)=\sum_{k=0}^{N} a_{k} \lambda^{k}
$$

So that the integral $J(T)$ is

$$
J_{k}\left(T_{j}\right)=\int_{0}^{\lambda_{c o}} W_{\lambda, T_{j}} \lambda^{k} d \lambda
$$

- Laguerre polynomials: those polynomials are defined as:

$$
L_{n}(x)=\frac{e^{x}}{n !} \frac{d^{n}}{d x^{n}}\left(e^{-x} x^{n}\right)
$$

In this case we have:

$$
S(\lambda)=\sum_{k=0}^{N} a_{k} L(k, x)
$$

The integral expression for the computation of the matrix elements will be:

$$
J_{k}\left(T_{j}\right)=\int_{0}^{\lambda_{c o}} W_{\lambda, T_{j}} L(k, x) d \lambda
$$


- Hermite polynomials: these polynomials are defined as:

$$
H_{n}(x)=(-1)^{n} e^{x^{2}} \frac{d^{n}}{d x^{n}} e^{-x^{2}}
$$

The integral expression for the computation of the matrix elements will be:

$$
J_{k}\left(T_{j}\right)=\int_{0}^{\lambda_{c o}} W_{\lambda, T_{j}} H(k, x) d \lambda
$$

- Sinc function: this function is defined as:

$$
\operatorname{Sinc}(x)= \begin{cases}\frac{\sin (\pi x)}{\pi x} & \text { if } x \neq 0 \\ 1 & \text { if } x=0\end{cases}
$$

The resolution of the Fredholm equation requires some manipulations of the function. In a finite interval $[a, b]$ it can be considered a basis function:

$$
z(x, k)=\frac{\sin \left(\frac{\pi(\mu(x)-k h)}{h}\right)}{\frac{\pi(\mu(x)-k h)}{h}}
$$

where:

$$
\mu(x)=\ln \left(\frac{x-a}{b-x}\right)
$$

And $h$ is the sample step. So, the function to be integrated in order to calculate the elements of the matrix $J(T)$ is:

$$
J_{k}\left(T_{j}\right)=\int_{0}^{\lambda_{c o}} \frac{\sin \left(\frac{\pi(\mu(x)-k h)}{h}\right)}{\frac{\pi(\mu(x)-k h)}{h}} W_{\lambda, T_{j}} d \lambda
$$

Considering the function Sinc, from its simmetry, the sum is from $-\mathrm{N}$ to $\mathrm{N}$ :

$$
S(\lambda)=\sum_{k=0}^{N} a_{k} z(x, k)
$$

\section{Materials and Methods}

The analysis has been carried out using Matlab $(R$. The first step has been that to simulate a photodetector output voltage measurement using a known spectral response. This allows to have a vector of elements that is just the output voltage to the photodetector. The function used is of the form:

$$
S(\lambda)=\frac{4}{625}\left(-\lambda^{4}+25 \lambda^{2}\right)
$$

The performance of this function is shown in Figure 2. The analysis has been carried out in the band $[0-5] \mu \mathrm{m}$. From the figure we notice that at the extremes of the band the spectral response is canceled. Also, if using this function for a higher wavelength it has negative values, there are currently no problems because it will only be used in the right band. Subsequently, we pass the calculation of the spectral response using the tension vector found by the previous simulation; this type of approach is allowed, as the actual voltage measurements are known. For the calculation of $S$, we proceeded in the manner seen in section 3 by evaluating the performance of the basic functions. Basically speaking we have a vector of tensions $V\left(T_{j}\right)$ obtained varying the black-body temperature, a metrics obtained integrating from (18) and unknown vector of coefficients of the series (16) in which now $f(x)=S(\lambda)$. 


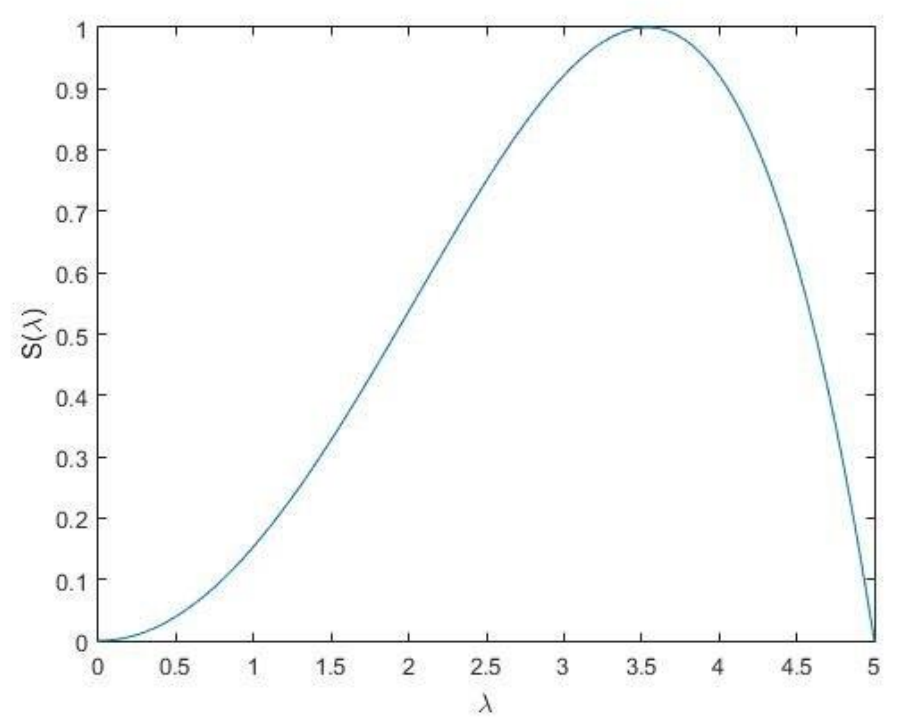

Figure 2. Theoretical spectral response used for simulations

\subsection{Power series approximation}

In this case it has been selected a power series for the basic function. The procedure has been described before so that it's possible to evaluate the performance in terms of system error. The error has been calculated computing the difference between the desired function and the reconstructed function. Other factors to be considered are the inversion of the matrix $J$ and the number of points to be used in the reconstruction. The analyses have been conducted with:

- $T=[500-700] K$

- $\lambda=[0-5] \mu m$

- $L=10 \mathrm{~cm}$

- $r_{0}=0.01 \mathrm{~cm}$

- $r=0.1 \mathrm{~cm}$

- $R_{\lambda}=10^{5} \mathrm{~V} / \mathrm{W}$

The parameter that has been varied was the number of points $N$, for values up to $N=7$ it's possible to rebuild the $S$ function very accurately, but with the increase of the number of points, Matlab $\mathbb{R}$ does not guarantee enough precision with the inverse matrix computation. In case of $N=7$ we can see how the function is estimated. Looking at 3 the curves are superimposed so it is possible to estimate the function with a maximum error in module of $6.7810^{-6}$. Increasing the number of points, this basis function no longer has the ability to accurately estimate the unknown function. Moreover, there are problems with the inverse matrix of $\mathrm{J}$ as its determinant is of the order of $10^{-53}$. Obviously, the error increases proportionally to the number of points. For example, in the case of $N=10$ we have a maximum error of 0.932 which is very high considering that the maximum of the function is 1 (Figure 3).

\subsection{Laguerre polynomials approximation}

In this case the basis functions are Laguerre polynomials. From (23) it's possible to note that even in this case it's possible to have problems similar to those seen with power series approximation. The simulation parameters were the same as before and we assessed how far we could increase the degree of the Laguerre polynomial. From the analyses carried out in such conditions, we can push to order polynomials up to $N=8$ with an error of 0.0013 (Figure 4). Moving to $N=9$ it's no longer possible to reconstruct the function and there are problems with the inverse of matrix $\mathrm{J}$ 


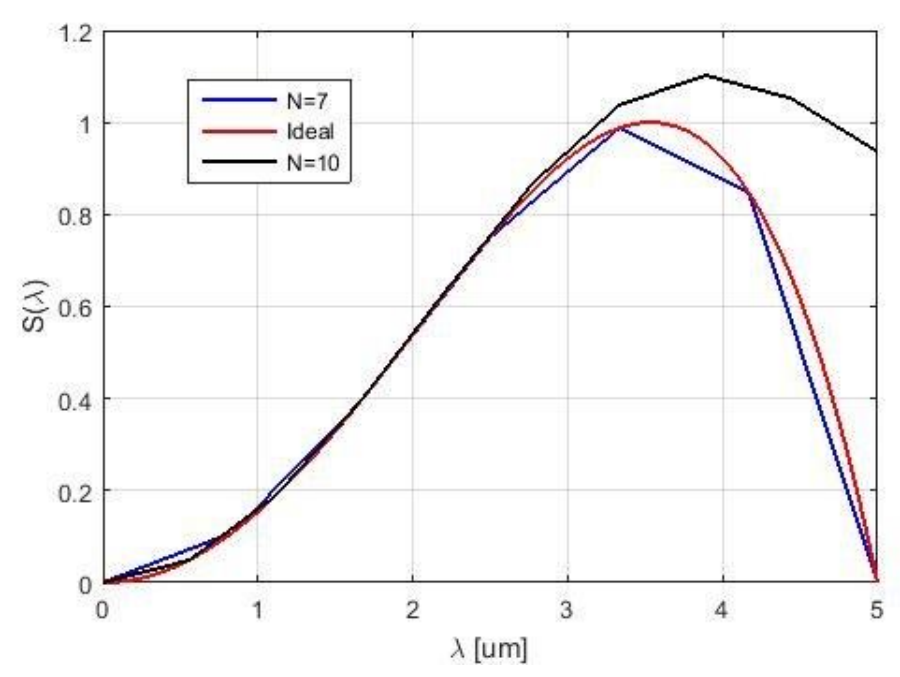

Figure 3. "S" Function reconstruction with Power Series

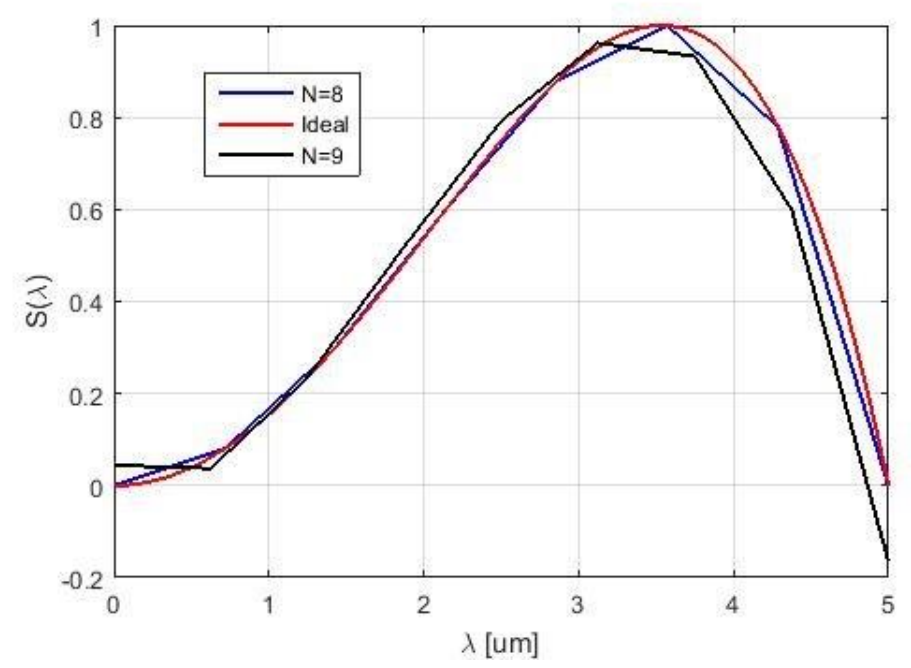

Figure 4. "S" Function reconstruction with Laguerre polynomials

\subsection{Hermite polynomials approximation}

The analysis has been carried out with the Hermite polynomials in formula (26). In this case the number of points is even lower in order not to have problems with the reconstruction and with the inversion of the matrix. In fact a number of points lower than $N=6$ have been used. Let's analyse the case of $N=5$. The ideal curve and the real curve are superimposed, so the reconstruction has been done correctly considering also that the error module is $6.23 * 10^{-11}$. By climbing the number of points and then the degree of polynomials, we have a good reconstruction of the function but there are problems with the inversion of $\mathrm{J}$, for $N=7$ (Figure 5).

\subsection{Approximation with "Sinc" functions}

For the estimation of the spectral response with the "Sinc" function, we need to make additional considerations since the basic function (29) seen in the previous section has problems at the extremes. In the interval $[0 \mu m-5 \mu m]$ the logarithm tends to minus infinite and therefore this is not a feasible situation, so we restricted the band considering a lower wavelength of $1 \mu \mathrm{m}$. As far as the upper extremity is concerned, in the cut-off frequency analysis we have the deletion of the denominator of 


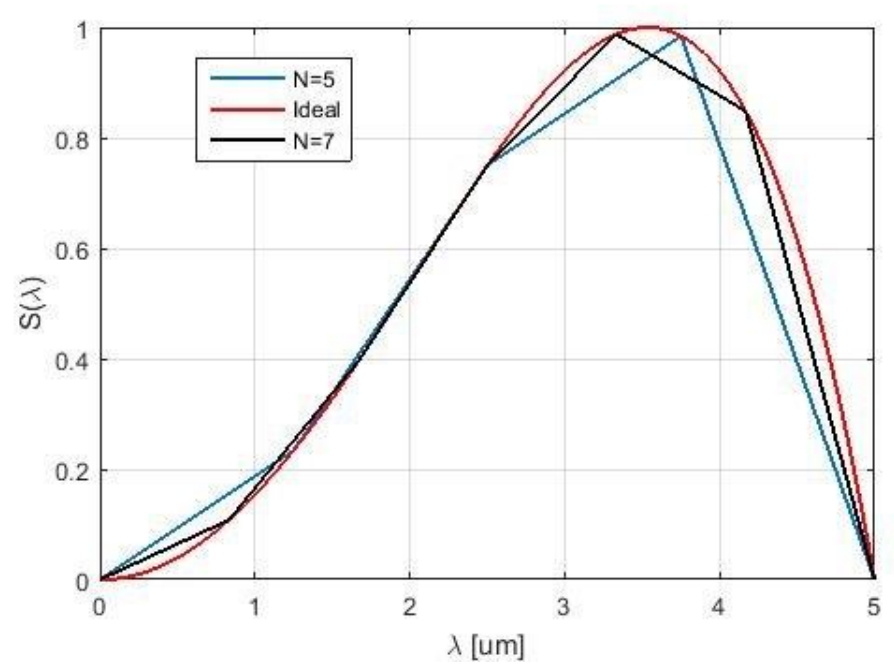

Figure 5. "S" Function reconstruction with Hermite polynomials

the fraction in the logarithm and therefore a useless value for the analysis. To this purpose, we shifted a small amount with respect to the cut-off frequency in order to avoid the zero in the denominator of the logarithm argument. In the case of the "Sinc" we have seen that the sum of the spectral response reconstruction goes from $-\mathrm{N}$ to $\mathrm{N}$, so the analysis is made on $2 \mathrm{~N}+1$ points. Let's see the case with $N=4$ (Figure 6) It can be noted that the function is reconstructed fairly faithfully up to the cut-off frequency where we find a deviation between the reconstructed and ideal spectral response. In this case, however, we have also the problem of the matrix $J(T)$ inversion. We propose the analysis with $N=2$ where we do not have the problem on the matrix but loose the maximum of function $S$ (Figure 6). Regarding the reconstruction it is done in a good way even if the reconstructed function deviates at some points from the ideal one.

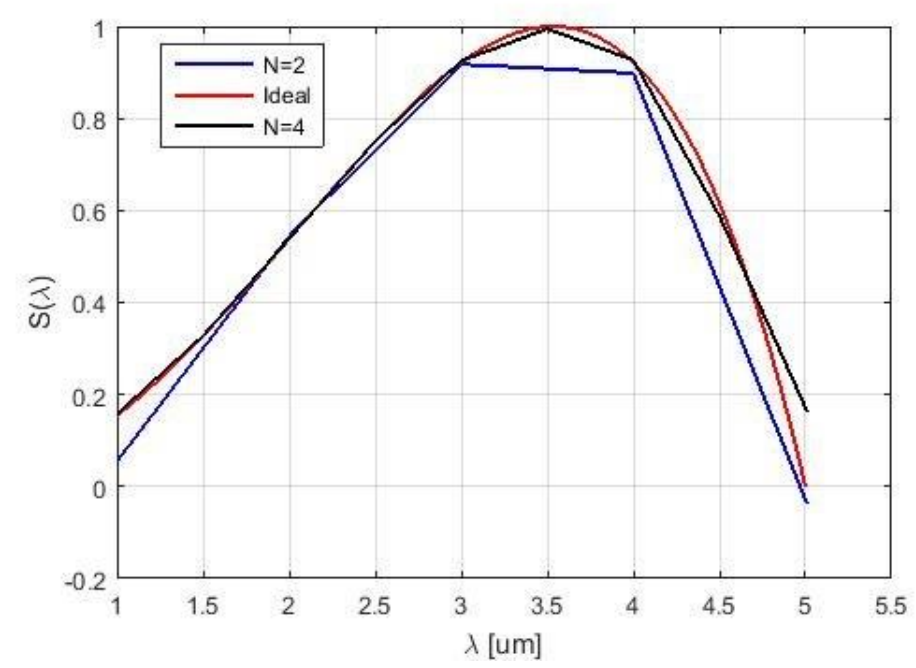

Figure 6. "S" Function reconstruction with Sinc functions

\section{Conclusions}

The proposed method is useful whenever the responsivity curve $S(\lambda)$ of a detector is unknown. We proposed several mathematical approaches to solve the Fredholm integral equation obtained varying the black body temperature. With this method we can get a rough approximation of the $S(\lambda)$ 
curve and so of the cut - off wavelength. The roughness of the spectral response estimation is depending on the number of measurement carried out. We applied this method using a simulated but realistic $S(\lambda)$ and we showed that it's possible to rebuild the function with a good level of approximation using only five or six measurements.

\section{Patents}

EU Patent EP2947435(A1) : Method For Estimating The Spectral Response Of An Infrared Photodetector US Patent US20150330837: Method For Estimating The Spectral Response Of An Infrared Photodetector IT Patent 0001423853: Metodo per la stima della risposta spettrale di un fotorilevatore ad infrarossi

Author Contributions: Conceptualization and Formal analysis, Riccardo Liberati and Massimiliano Rossi; Software and Writing-original draft, Alessio del Paggio

Funding: This research received no external funding

Conflicts of Interest: The authors declare no conflict of interest

\section{Abbreviations}

The following abbreviations are used in this manuscript:

2D Two-dimensional

NETD Noise-equivalent temperature

\section{References}

1. Michael A. Kinch, Fundamentals of Infrared Detector Materials, SPIE Publications, November 2007.

2. Willardson and Beer, Semiconductors and Semimetals Vol 12:Infrared Detector II, Academic Press, December 1977.

3. J. M. Lloyd, Thermal Imaging Systems, Springer US 1975.

4. R. H. Kingston, Detection of Optical and Infrared Radiation, Springer Berlin Heidelberg, 1978.

5. William L. Wolfe, Infrared Design Examples, SPIE Publications, July 1999.

6. William L. Wolfe, Introduction to Infrared System Design, SPIE Publications, March 1996.

7. Cornelius J. Willers, Electro-Optical System Analysis and Design: A Radiometry Perspective, SPIE Publications, 2013. 